\title{
GaN-HEMT Dynamic ON-state Resistance characterisation and Modelling
}

\author{
Ke Li, Paul Evans, Mark Johnson \\ Power Electronics, Machine and Control group \\ University of Nottingham, UK \\ Email:ke.li@nottingham.ac.uk, paul.evans@nottingham.ac.uk, mark.johnson@nottingham.ac.uk
}

\begin{abstract}
GaN-HEMTs suffer from trapping effects which might increase device $\mathrm{ON}$-state resistance $\left(R_{\mathrm{DS} \text { (on })}\right)$ values. Thus, dynamic $R_{\mathrm{DS} \text { (on) }}$ of a commercial GaN-HEMT is characterized at different bias voltages in the paper by a proposed measurement circuit. Based on the measurement results, a behavioural model is proposed to represent device dynamic $R_{\mathrm{DS}(\text { on) }}$ values, in which trapping and detrapping time constant is represented by a series of RC network. The model is simulated in PSPICE, of which the simulation results of $R_{\mathrm{DS}(\mathrm{on})}$ values are compared and validated with the measurement when device switches in a power converter with different duty cycles and switching voltages. The results show that $R_{\mathrm{DS}(\text { on) }}$ values of this device would increase due to trapping effects.
\end{abstract}

Keywords-GaN-HEMT; Dynamic ON-state resistance; Power semiconductor device characterisation; Power semiconductor device modelling; Behavioural model

\section{INTRODUCTION}

Application of GaN power semiconductor devices is becoming more and more popular in power electronics systems design, because GaN devices can operate at high temperature, switch fast and produce low power loss. In order to better use GaN devices in power electronics systems, it is necessary to understand their characteristics. High-ElectronMobility Transistors (HEMTs) are the most widely used $\mathrm{GaN}$ power electronic devices, but they suffer from electron trapping effects that reduce their current carrying capability [1], [2].

Illustrated in Fig. 1, this trapping effect is related mainly with two effects when device in OFF-state, one is the bias $V_{\mathrm{DS}}$ voltage value and another is the bias time (trapping time), which would give rise to the increase of $\mathrm{GaN}$ device $\mathrm{ON}$-state resistance $\left(R_{\mathrm{DS}(\mathrm{on})}\right)$ value. According to the results presented by authors in [3], GaN device $R_{\mathrm{DS}(\text { on) }}$ values would increase by a maximal factor of 4 after $1 \mathrm{~ms}$ bias time depending on bias $V_{\mathrm{DS}}$ voltage value. The results presented by authors in [4] demonstrate that device $R_{\mathrm{DS}(\text { on })}$ values would further increase $50 \%$ if bias time is doubled. In the on-state (low bias), detrapping occurs and the $R_{\mathrm{DS}(\text { on) }}$ values decrease to the steady state value at a rate characterised by the detrapping time. As shown in [3], GaN device dynamic $R_{\mathrm{DS}(\mathrm{on})}$ values would decrease $30 \%$ after $10 \mu$ s detrapping time.

When employing GaN transistors in power electronics circuits, GaN device might switch with different periods and duty

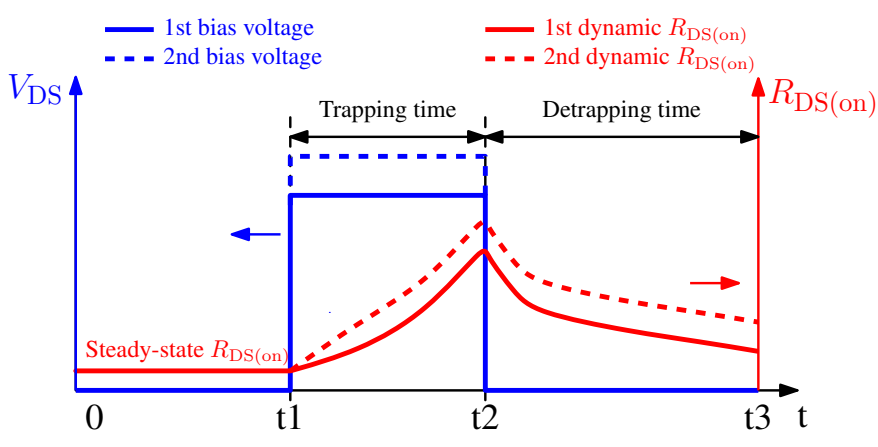

Fig. 1: GaN-HEMT dynamic $R_{\mathrm{DS}(\text { on) }}$ values due to trapping effects

cycles leading to a combination of trapping and detrapping effects and consequently uncertainty in the actual value of $R_{\mathrm{DS}(\mathrm{on})}$. This will lead to uncertainty in device power loss, making predictions of converter efficiency and cooling system design challenging. The ability to characterise and model GaNHEMT $R_{\mathrm{DS}(\text { on) }}$ values is thus an important design consideration.

There are mainly two different methods to measure GaNHEMT dynamic $R_{\mathrm{DS}(\mathrm{on})}$ values: one method is by using directly a measurement equipment [4], and another one is by using an electrical circuit, where different circuit topologies are proposed by authors in [3], [5]-[8]. In this paper, a new characterisation circuit is presented to measure $\mathrm{GaN}$ HEMT dynamic $R_{\mathrm{DS}(\text { on) }}$ values, which could be easily implemented. Compared to the above different circuits, this new measurement circuit needs fewer components and offers an alternative method to characterise the device and to compare the results. Based on the dynamic $R_{\mathrm{DS} \text { (on) }}$ measurement results, a behavioural model is thus proposed to present device trapping and detrapping effect, which could be used in a circuit simulator.

The paper has the following structure. First the measurement circuit to characterize GaN-HEMT dynamic $R_{\mathrm{DS}(\text { on) }}$ values is presented. Afterwards, measurement results of different trapping and detrapping time on $R_{\mathrm{DS}(\text { on) }}$ values are shown. Based on the measurement results, a behavioural model is proposed to represent device dynamic $R_{\mathrm{DS}(\text { on) }}$ values. The model is further validated by comparing with the measurement 
when device switches in a power converter. Conclusions are given at last.

\section{GAN-HEMT DYNAMIC ON-STATE RESISTANCE MEASUREMENT}

\section{A. Measurement circuit}

GaN device $R_{\mathrm{DS} \text { (on) }}$ values can be obtained by measuring device ON-state voltage $V_{\mathrm{DS}(\mathrm{on})}$ across it and current $I_{\mathrm{D}}$ through it in an electrical circuit. As the measured bias voltage when device is $\mathrm{OFF}\left(V_{\mathrm{DS} \text { (off) }}\right)$ could achieve more than several hundred times higher than device $V_{\mathrm{DS}(\mathrm{on})}$, a voltage clamping circuit is necessary to reduce the measured $V_{\mathrm{DS} \text { (off) }}$ in order to increase measurement accuracy. For this reason, the measurement circuit shown in Fig. 2 is constituted by a bias circuit to control device trapping time when it is OFF and a voltage clamping circuit to measure device $V_{\mathrm{DS}(\text { on })}$ value when it is $\mathrm{ON}$.

In the bias circuit, a transistor T1 is used to control DUT trapping time. A resistive load $R_{\text {load }}$ is used to set the current level when DUT is in ON-state. Because of the parasitic inductance $L_{\text {para }}$ of the $R_{\text {load }}$, two diodes D1, D2 offer a free wheeling path of the current when either T1 or DUT is switched from ON to OFF.

The voltage clamping circuit is constituted by a depletion mode (D-mode) Si-MOSFET and a zener diode. DUT measurement voltage $V_{\mathrm{DS}(\mathrm{m} \text {.) }}$ is measured across the zener diode. The principle of the voltage clamping circuit is that when DUT is ON, D-mode Si-MOSFET is in ON-state $\left(V_{\mathrm{g}_{\mathrm{m}} \mathrm{m}}\right.$ is superior to MOSFET threshold voltage $V_{\mathrm{th}}$ ), so points $s_{\mathrm{m}}$ and $d_{\mathrm{m}}$ are almost in the same potential and DUT $V_{\mathrm{DS}(\mathrm{on})}$ can thus be measured directly $\left(V_{\mathrm{DS}(\mathrm{m} .)}=V_{\mathrm{DS}(\mathrm{on})}\right)$. When DUT is OFF, zener diode junction capacitance is charged at first, so $V_{\mathrm{DS}(\mathrm{m} \text {.) }}$ increases to the zener diode clamping voltage $V_{\text {clamp }}$, and then $V_{\mathrm{g}_{\mathrm{m} \mathrm{s}}}$ is inferior to $V_{\mathrm{th}}$. Afterwards, D-mode MOSFET is pinched OFF and its inter-electrode capacitance $C_{\mathrm{d}_{\mathrm{m}} \mathrm{m}}$ is charged to withstands almost the whole bias $V_{\mathrm{DS}}$ voltage $\left(V_{\mathrm{DS}} \gg V_{\mathrm{DS}(\mathrm{m} .)}\right)$. It is to be noted that as there is a leakage current balance between D-mode MOSFET and Zener diode, $V_{\mathrm{DS}(\mathrm{m} .)}$ is inferior to $V_{\text {clamp }}$ in steady-state. Instead of measuring voltage range between $V_{\mathrm{DS}(\mathrm{on})}$ and $V_{\mathrm{DS}}$, a much smaller voltage range between $V_{\mathrm{DS} \text { (on) }}$ and $V_{\text {clamp }}$ is measured, thus the measurement sensitivity is increased. Compared to the similar type voltage clamping circuits that are analyzed by authors in [6], less components and no external power supply are used in this clamping circuit.

Device steady-state $R_{\mathrm{DS}(\text { on) }}$ could be measured by applying the control signal shown in Fig. 2b, where DUT is kept always in ON-state and T1 is controlled by a single pulse.

Device dynamic $R_{\mathrm{DS}(\mathrm{on})}$ could be measured by applying the control signal shown in Fig. 2c, where DUT is initially kept in ON-state and T1 blocks all the bias voltage. Then at $\mathrm{t} 1$, DUT is switched OFF and at $\mathrm{t} 2, \mathrm{~T} 1$ is switched ON, thus

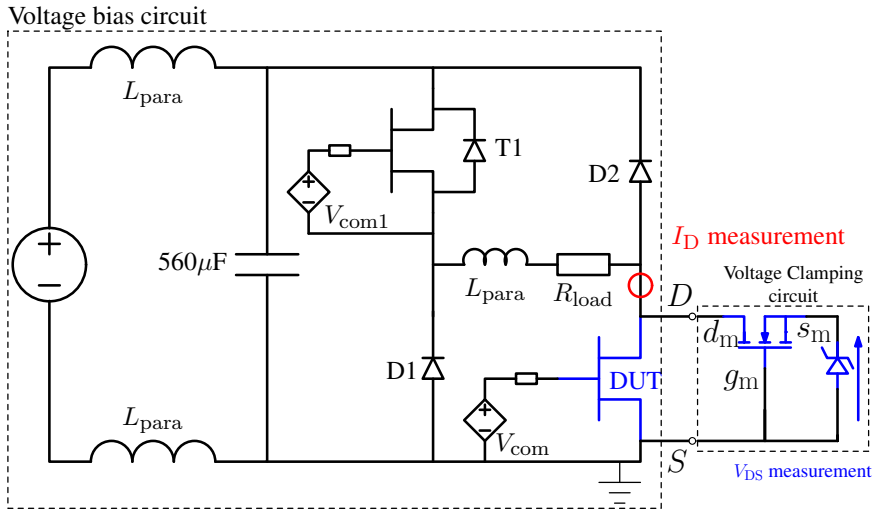

(a) Measurement configuration

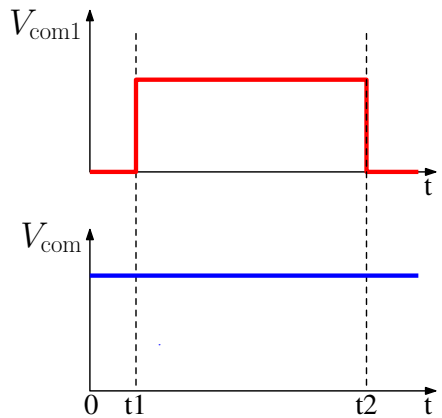

(b) Control signal to measure steadystate $R_{\mathrm{DS} \text { (on) }}$

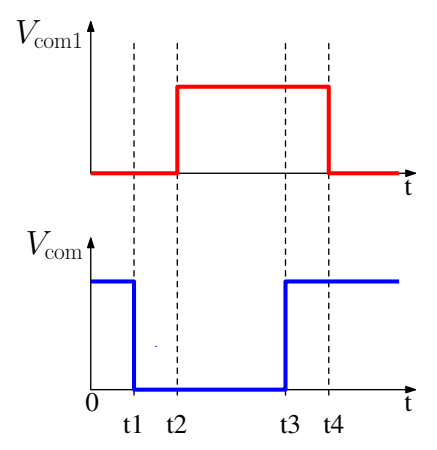

(c) Control signal to measure dynamic $R_{\mathrm{DS} \text { (on) }}$
Fig. 2: GaN-HEMT dynamic ON-state resistance measurement circuit and control signals

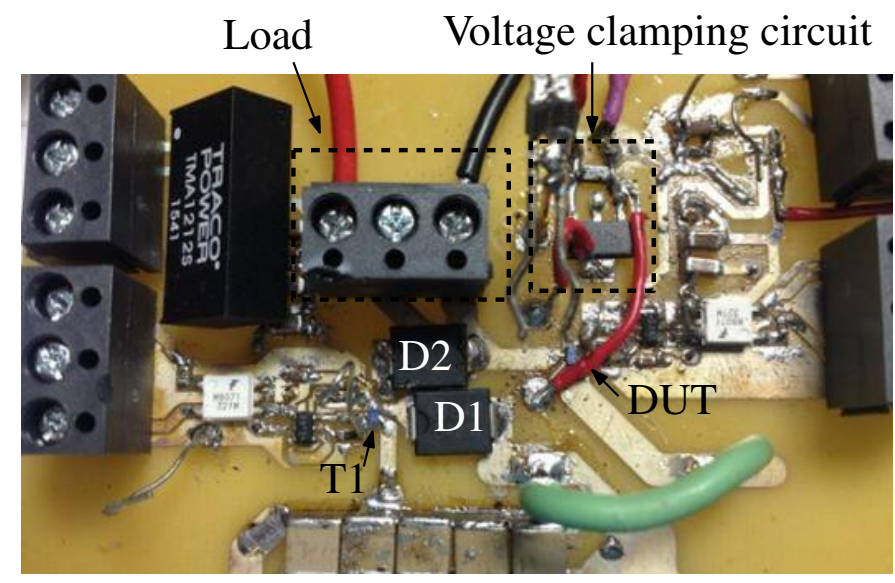

Fig. 3: Realization of the measurement circuit

all the bias voltage is across DUT. Afterwards, at t3, DUT is switched ON again, so current $I_{\mathrm{D}}$ flows through the DUT. Finally at $\mathrm{t} 4$, T1 is switched OFF. Thus, DUT trapping time is controlled by $\mathrm{t} 2-\mathrm{t} 3$ while detrapping time is controlled by t3-t4, so $R_{\mathrm{DS}(\mathrm{on})}$ values of different trapping and detrapping time could be measured.

The realization of the measurement circuit is shown in Fig. 3. In the measurement, $R_{\text {load }}=100 \Omega$, T1 is a commercial GaN-HEMT (EPC2012C, 200V/5A) while D1 and D2 are the 

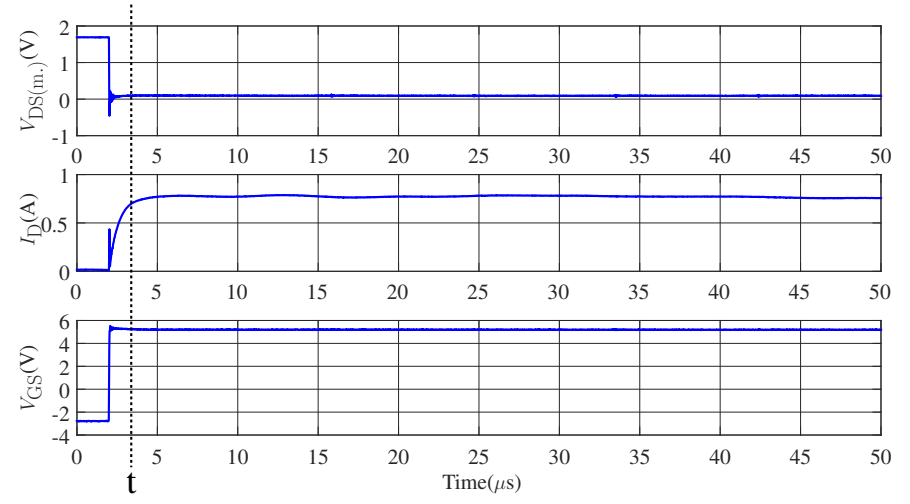

Fig. 4: Measured waveforms when device is bias at $80 \mathrm{~V}$ for $10 \mu \mathrm{s}$

same Schottky diode (MBRS4201T3G, 200V/4A). Dynamic $R_{\mathrm{DS}(\mathrm{on})}$ values of a DUT, which is the same as $\mathrm{T} 1$, is measured by the above circuit, of which the results are presented in the next section.

\section{B. Measurement results}

Several major parameters of the measurement equipments and clamping circuit devices are summarized in TABLE. I. In the measurement, the maximal measured $V_{\mathrm{DS}}$ voltage is $3.3 \mathrm{~V}$, which could achieve a measurement accuracy of at least $\frac{3.3}{2^{8}}=0.013 \mathrm{~V}$ by using a 8 -bit resolution oscilloscope. GaNHEMT dynamic $R_{\mathrm{DS}(\mathrm{on})}$ values are measured when it is biased at $80 \mathrm{~V}$ and $120 \mathrm{~V}$.

When GaN-HEMT is biased at $80 \mathrm{~V}$ and trapping time is $10 \mu \mathrm{s}$, the measured voltage $V_{\mathrm{DS}(\mathrm{m} \text {.) }}$, current $I_{\mathrm{D}}$ and voltage $V_{\mathrm{GS}}$ are shown in Fig. 4. Because of the voltage clamping circuit, $V_{\mathrm{DS}(\mathrm{m} \text {.) }}$ is about $1.7 \mathrm{~V}$ when DUT is OFF, which is much smaller than the bias voltage $(80 \mathrm{~V})$. It is to be noted that maximal $V_{\mathrm{DS}(\mathrm{m} .)}$ almost does not change when the bias voltage increases to $120 \mathrm{~V}$, thus the measurement accuracy is improved in comparison to a direct measurement.

DUT dynamic $R_{\mathrm{DS}(\text { on) }}$ values are calculated after instant $t$ in Fig. 4, which is $1 \mu$ s after OFF-ON transition when each electrical parameter stabilizes. The obtained $R_{\mathrm{DS}(\text { on) }}$ values based on the above waveforms is shown in Fig. 5, in which $R_{\mathrm{DS}(\text { on) }}$ values are compared between steady-state and dynamic-state when device is biased at $80 \mathrm{~V}$ and $120 \mathrm{~V}$ at $25^{\circ} \mathrm{C}$.

As shown in the results, $R_{\mathrm{DS} \text { (on) }}$ values increase with the trapping time and it decreases with detrapping time, and it increases more with a higher bias voltage. For this device, it is observed that when device biased by a certain trapping time, it needs a longer detrapping time to reduce its dynamic $R_{\mathrm{DS}(\text { on) }}$ values to the steady-state values, which shows that effective $R_{\mathrm{DS}(\text { on) }}$ values are likely to be higher than theoretical values in application. A behavioural model is proposed based on the measurement results, which will be presented in the next section.

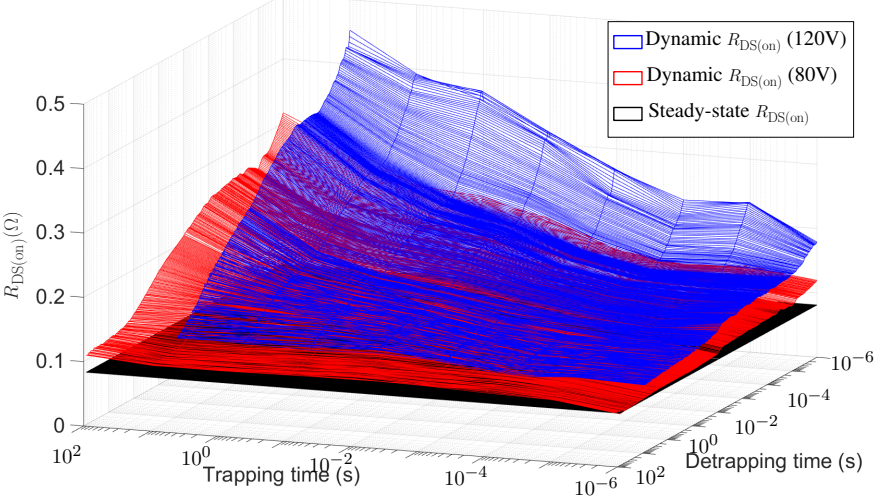

Fig. 5: Comparison of steady-state $R_{\mathrm{DS}(\mathrm{on})}$ and dynamic $R_{\mathrm{DS}(\text { on) }}$ at $25^{\circ} \mathrm{C}$

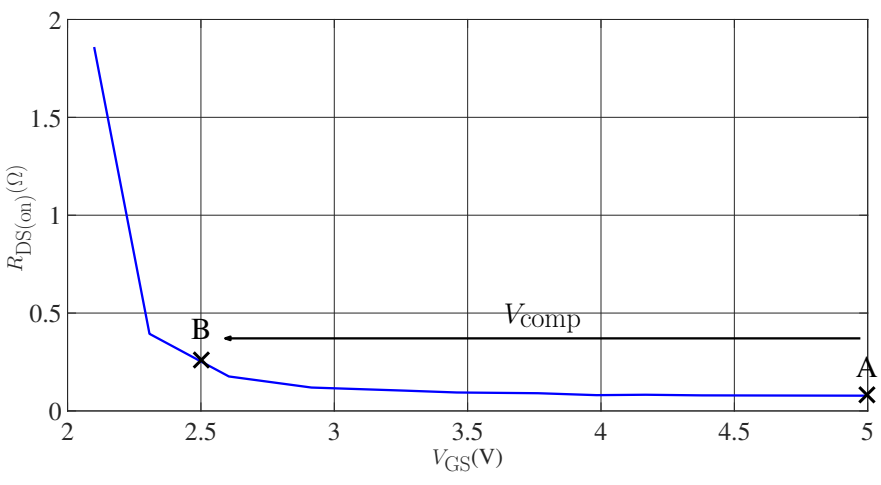

Fig. 6: $R_{\mathrm{DS}(\mathrm{on})}$ values of different $V_{\mathrm{GS}}$ voltages at $25^{\circ} \mathrm{C}$

\section{GAN-HEMT DYNAMIC ON-STATE RESISTANCE MODELLING}

\section{A. Behavioural model}

When in steady-state, the measured device $R_{\mathrm{DS}(\text { on) }}$ values of different $V_{\mathrm{GS}}$ voltages at $25^{\circ} \mathrm{C}$ is shown in Fig. 6, which presents that $R_{\mathrm{DS}(\mathrm{on})}$ values increase when $V_{\mathrm{GS}}$ voltage decreases.

According to this $R_{\mathrm{DS}(\text { on) }}-V_{\mathrm{GS}}$ relation, the obtained dynamic $R_{\mathrm{DS}(\text { on) }}$ values could be represented by static $R_{\mathrm{DS}(\text { on) }}$ values at the equivalent gate voltage shown in Fig. 6, where point A corresponds to the $R_{\mathrm{DS}(\text { on) }}$ value when it is obtained at steady-state, and point $\mathrm{B}$ corresponds to the $R_{\mathrm{DS}(\mathrm{on})}$ value after certain trapping time. The $V_{\mathrm{GS}}$ voltage difference between point $\mathrm{A}$ and point $\mathrm{B}$, which is defined as $V_{\text {comp }}$, could be applied to represent $R_{\mathrm{DS}(\text { on })}$ change during the trapping and detrapping process. After adding $V_{\text {comp }}$ in gate circuit, which is shown in Fig. 7, device $V_{\mathrm{GS}}$ voltage $\left(V_{\mathrm{GS}}=V_{\mathrm{G}}-V_{\text {comp }}\right)$ after trapping time could be adjusted, thus a dynamic $R_{\mathrm{DS}(\text { on) }}$ value could be obtained.

It is shown that $V_{\text {comp }}$ increases with the trapping time and decreases with the detrapping time, in which the phenomena could be modelled in the form of an RC circuit, which is presented in Fig. 7. In each RC unit, $V_{\text {compn }}$ increases when 


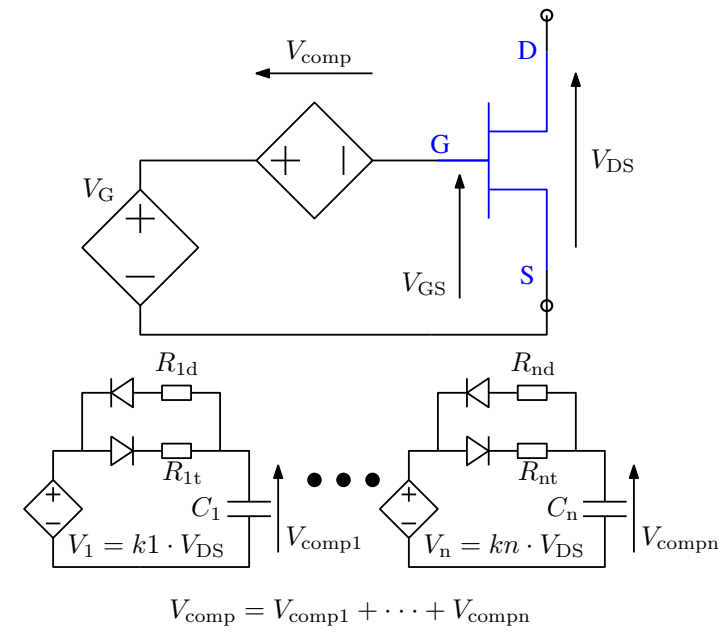

Fig. 7: Dynamic $R_{\mathrm{DS}(\mathrm{on})}$ values representation by behavioural model

capacitor $C_{\mathrm{n}}$ is charged by a controlled voltage source $V_{\mathrm{n}}$ through resistor $R_{\mathrm{nt}}$ and it decreases when $C_{\mathrm{n}}$ is discharged through resistor $R_{\text {nd }}$. Thus, GaN-HEMT trapping and detrapping effect could be represented by a series of the RC units, and $V_{\text {comp }}$ value is the sum of the voltage of the capacitor in each unit. Based on the measurement results shown in Fig. 5 when bias voltage is $80 \mathrm{~V}$, seven $\mathrm{RC}$ units are used to represent device dynamic $R_{\mathrm{DS}(\text { on) }}$ values, in which parameters $V_{1}-V_{7}$, $C_{1}-C_{7}, R_{1 \mathrm{~d}}-R_{7 \mathrm{~d}}$ and $R_{1 \mathrm{t}}-R_{7 \mathrm{t}}$ are obtained by fitting methods. The values of the above parameters are given in TABLE. II. The comparison between the model and the measurement is shown in Fig. 8, where the model represents generally well dynamic $R_{\mathrm{DS}(\mathrm{on})}$ values of different trapping and detrapping time.

After obtaining the above parameters, the model illustrated in Fig. 7 could be easily implemented in a circuit simulator. In this paper, the model is implemented in PSPICE, where $V_{\text {comp }}$ and $V_{1} \cdots V_{7}$ are represented by voltage controlled voltage source and coefficients $k_{1} \cdots k_{7}$ could be calculated once $V_{1} \cdots V_{7}$ are known.

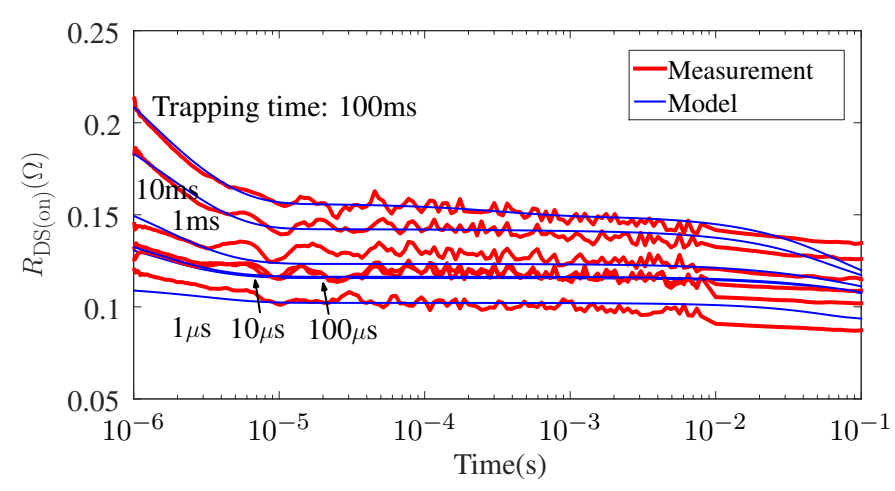

Fig. 8: Comparison between the measurement and model on dynamic $R_{\mathrm{DS}(\text { on) }}$ values as a function of detrapping time for different trapping times when $V_{\mathrm{DS}}=80 \mathrm{~V}$

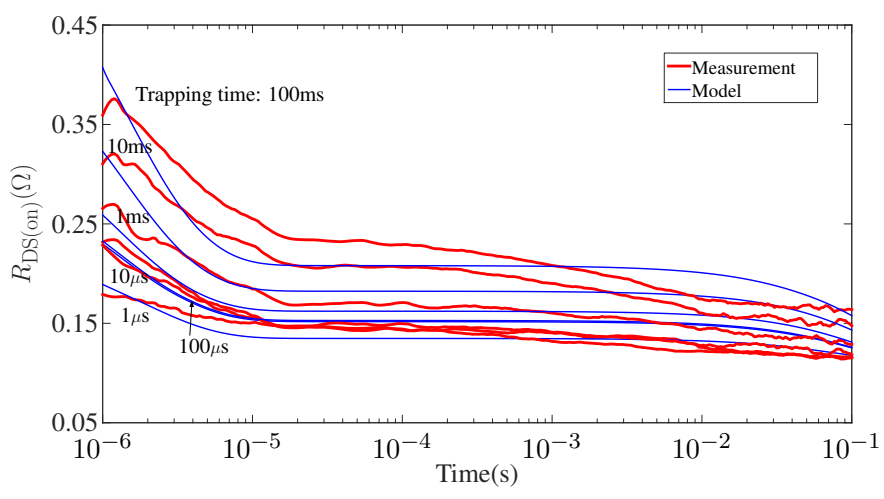

Fig. 9: Comparison between the measurement and model on dynamic $R_{\mathrm{DS}(\text { on) }}$ values when $V_{\mathrm{DS}}=120 \mathrm{~V}$

\section{B. Model extension for different bias voltage}

Following by that, the same model is used to represent the measurement results when the device is biased at $V_{\mathrm{DS}}=120 \mathrm{~V}$. In order to represent device dynamic $R_{\mathrm{DS}(\mathrm{on})}$ values of different bias voltages by the model and easily implement it, only $V_{1} \ldots V_{7}$ are fitted again when $V_{\mathrm{DS}}=120 \mathrm{~V}$, while the other parameters are the same as obtained when $V_{\mathrm{DS}}=80 \mathrm{~V}$.

In such a case, bigger $V_{\text {comp }}$ values might be obtained, so

TABLE I: Major parameters of the measurement equipments and clamping circuit devices

\begin{tabular}{|c|c|c|c|c|}
\hline Oscilloscope & Current probe & Voltage probe & D-mode MOSFET & Zener diode \\
\hline DPO4104B (1GHz, 8-bit) & TCP312 (100MHz, 30A) & TPP1000 $(1 \mathrm{GHz}, 300 \mathrm{~V})$ & BSP149 (200V, $\left.V_{\mathrm{th}} \approx-1.4 \mathrm{~V}\right)$ & BZT52C3V3 (3.3V) \\
\hline
\end{tabular}

TABLE II: Parameters using to represent GaN-HEMT trapping effect when device is bias at $80 \mathrm{~V}$

\begin{tabular}{|c|c|c|c|c|c|c|}
\hline$V_{1}$ & $V_{2}$ & $V_{3}$ & $V_{4}$ & $V_{5}$ & $V_{6}$ & $V_{7}$ \\
\hline $0.51 \mathrm{~V}$ & $0.6 \mathrm{~V}$ & $0.28 \mathrm{~V}$ & $0.25 \mathrm{~V}$ & $0.66 \mathrm{~V}$ & $0.1 \mathrm{~V}$ & $0.97 \mathrm{~V}$ \\
\hline$R_{1 \mathrm{~d}}$ & $R_{2 \mathrm{~d}}$ & $R_{3 \mathrm{~d}}$ & $R_{4 \mathrm{~d}}$ & $R_{5 \mathrm{~d}}$ & $R_{6 \mathrm{~d}}$ & $R_{7 \mathrm{~d}}$ \\
\hline $2.5 \times 10^{9} \Omega$ & $8.5 \times 10^{5} \Omega$ & $2.7 \times 10^{3} \Omega$ & $1 \times 10^{12} \Omega$ & $1 \times 10^{12} \Omega$ & $1 \times 10^{14} \Omega$ & $1 \times 10^{10} \Omega$ \\
\hline$R_{1 \mathrm{t}}$ & $R_{2 \mathrm{t}}$ & $R_{3 \mathrm{t}}$ & $R_{4 \mathrm{t}}$ & $R_{5 \mathrm{t}}$ & $R_{6 \mathrm{t}}$ & $R_{7 \mathrm{t}}$ \\
\hline $85.6 \Omega$ & $5.6 \Omega$ & $31.5 \Omega$ & $2 \times 10^{6} \Omega$ & $1.6 \times 10^{4} \Omega$ & $2.9 \times 10^{7} \Omega$ & $0.05 \Omega$ \\
\hline$C_{1}$ & $C_{2}$ & $C_{3}$ & $C_{4}$ & $C_{5}$ & $C_{6}$ & $C_{7}$ \\
\hline $1.9 \times 10^{-9} \mathrm{~F}$ & $1.3 \times 10^{7} \mathrm{~F}$ & $1 \times 10^{-9} \mathrm{~F}$ & $8.6 \times 10^{-10} \mathrm{~F}$ & $2.8 \times 10^{-11} \mathrm{~F}$ & $2.2 \times 10^{-9} \mathrm{~F}$ & $9.4 \times 10^{-11} \mathrm{~F}$ \\
\hline
\end{tabular}




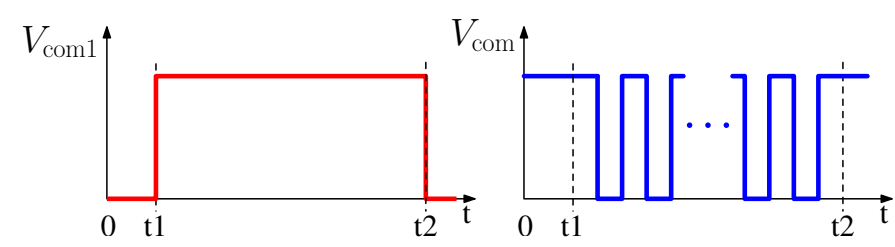

Fig. 10: Control signal when device switches continuously in a power converter

bigger dynamic $R_{\mathrm{DS}(\text { on) }}$ values would be represented when device is biased at the same trapping time as $V_{\mathrm{DS}}=80 \mathrm{~V}$. It corresponds to the measurement results shown in Fig. 5 that bigger dynamic $R_{\mathrm{DS}(\text { on) }}$ values are obtained when device is bias at $120 \mathrm{~V}$ than at $80 \mathrm{~V}$.

Obviously, all the above 28 parameters could be fitted again to represent the measurement results when $V_{\mathrm{DS}}=120 \mathrm{~V}$. However, it might be difficult to find the trend of each parameter with the bias voltage, especially that of $R_{\mathrm{nd}}, R_{\mathrm{nt}}$ and $C_{\mathrm{n}}$. In contrary, the trend of $V_{\mathrm{n}}$ with the bias voltage is easier to be found and implemented in the model.

The obtained parameters of $V_{1}-V_{7}$ in this situation are given in TABLE III. The comparison between the model and the measurement when device biased at $120 \mathrm{~V}$ is shown in Fig. 9, where it is shown that the model could represent the measurement in a reasonable way.

As GaN-HEMT suffered from trapping effect, its $R_{\mathrm{DS} \text { (on) }}$ values might increase when it switches continuously in a power converter. For this reason, $R_{\mathrm{DS}(\mathrm{on})}$ values estimated by the above model is compared with the measurement, and the results will be presented in the next section.

\section{Model Validation}

\section{A. Switching voltage at $80 \mathrm{~V}$}

The same electrical circuit shown in Fig. 2a with the control signal shown in Fig. 10 is used to measure device dynamic $R_{\mathrm{DS}(\text { on) }}$ values when it switches continuously. In order to avoid device self heating, it switches at $10 \mathrm{kHz}$ with different duty cycles and a duration of $0.1 \mathrm{~s}$.

When switching voltage is $80 \mathrm{~V}$ and duty cycle (D) is $50 \%$ and $90 \%$, the comparison of the $R_{\mathrm{DS}(\text { on) }}$ values is shown in Fig. 11a and in Fig. 11b separately. As explained previously, device detrapping time to reach its steady-state $R_{\mathrm{DS}(\text { on) }}$ value is longer than trapping time to increase its dynamic $R_{\mathrm{DS}(\mathrm{on})}$ values, which explains that the measured $R_{\mathrm{DS} \text { (on) }}$ values increase to a factor of two higher than its steady-state value after $0.1 \mathrm{~s}$ in the measurement. The trapping time in the above measurements varies from $50 \mu$ s to $10 \mu \mathrm{s}$ and detrapping time

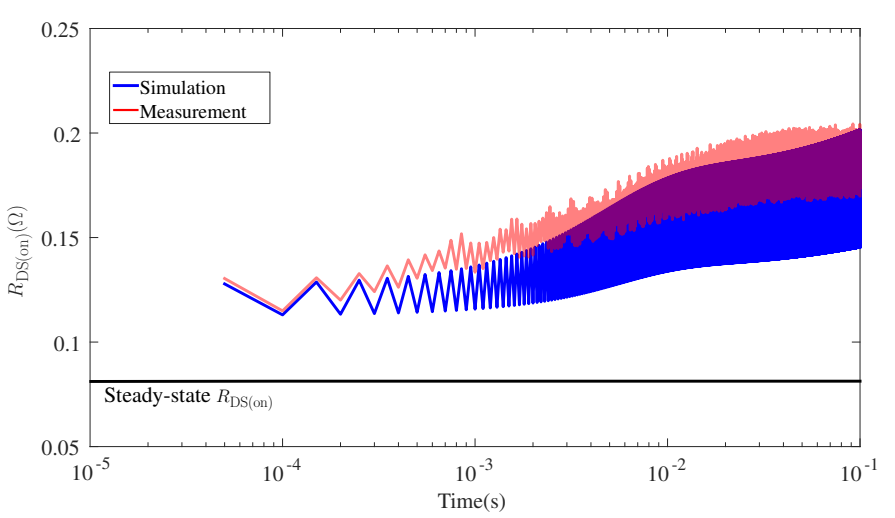

(a) Duty cycle is $50 \%$

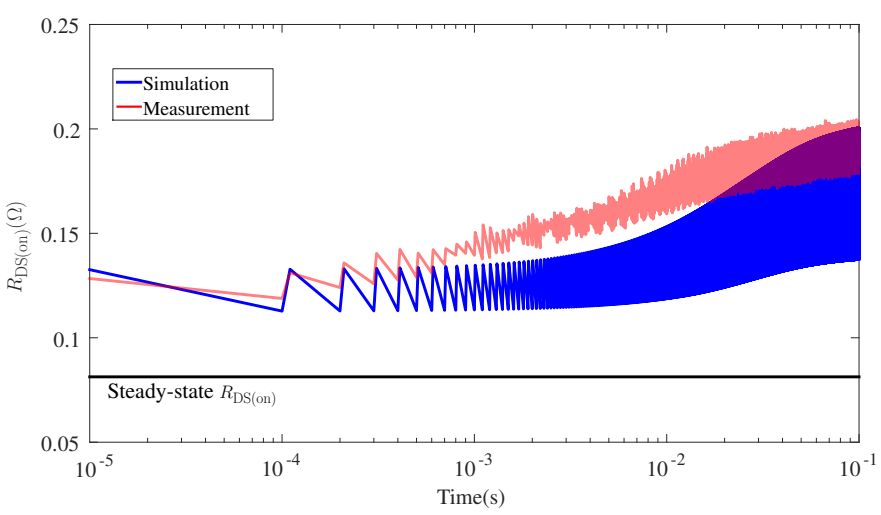

(b) Duty cycle is $90 \%$

Fig. 11: Comparison between the measurement and simulation on $R_{\mathrm{DS}(\text { on) }}$ values when device switches at $80 \mathrm{~V}$

varies from $50 \mu \mathrm{s}$ to $90 \mu \mathrm{s}$. According the measurement results shown in Fig. 8, dynamic $R_{\mathrm{DS}(\text { on) }}$ values are not influenced by detrapping time obviously in this detrapping time range, which might explain that a change of duty cycle does not change device $R_{\mathrm{DS}(\mathrm{on})}$ values obviously. The trapping effects can increase $R_{\mathrm{DS}(\text { on) }}$ values very quickly when trapping time inferior to $10 \mu \mathrm{s}$, thus it might still increase device $R_{\mathrm{DS}(\text { on) }}$ values when device using in high frequency converter (more than $100 \mathrm{kHz}$ ). However, if device switches in high frequency, the switching loss might increase device junction temperature $T_{\mathrm{j}}$, which would also increase device $R_{\mathrm{DS}(\text { on) }}$ values.

It is also shown in Fig. 11 that the trend of the $R_{\mathrm{DS}(\text { on) }}$ values increase in the measurement is represented generally well by the simulation, where the difference between the measurement and the simulation in both situations is within $17 \%$. The difference between the simulation and the measurement might be due to the difference between the model and the measurement shown in Fig. 8 on dynamic $R_{\mathrm{DS}(\text { on) }}$ values.

TABLE III: Parameters using to represent GaN-HEMT trapping effect when device is bias at $120 \mathrm{~V}$

\begin{tabular}{|c|c|c|c|c|c|c|}
\hline$V_{1}$ & $V_{2}$ & $V_{3}$ & $V_{4}$ & $V_{5}$ & $V_{6}$ & $V_{7}$ \\
\hline $0.143 \mathrm{~V}$ & $0.1 \mathrm{~V}$ & $0.32 \mathrm{~V}$ & $0.1 \mathrm{~V}$ & $0.74 \mathrm{~V}$ & $0.1 \mathrm{~V}$ & $1.59 \mathrm{~V}$ \\
\hline
\end{tabular}




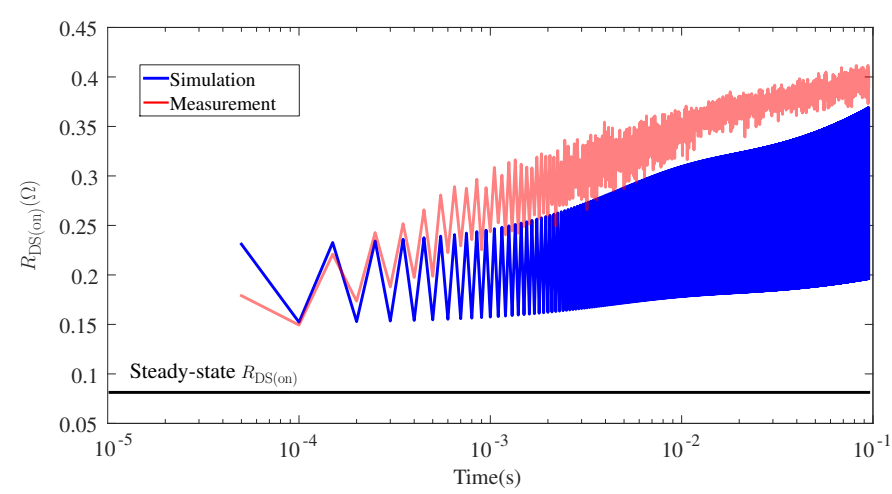

(a) Device switches at $120 \mathrm{~V}(\mathrm{D}=50 \%)$

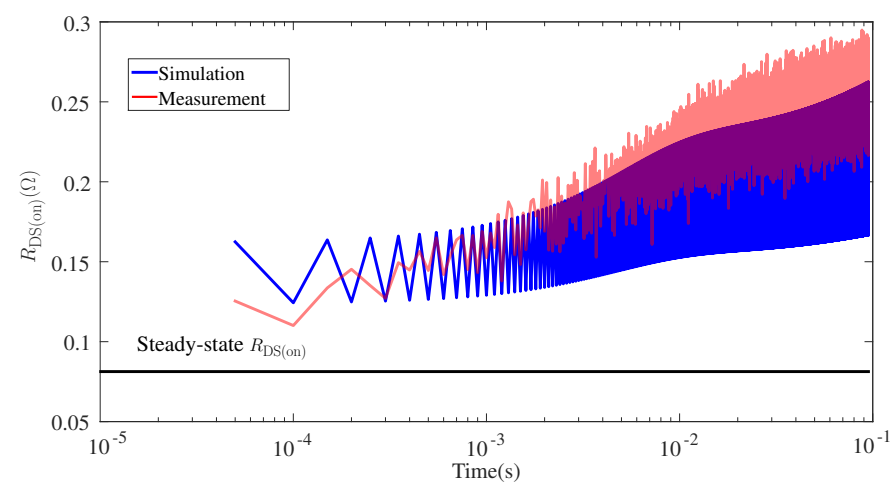

(b) Device switches at $100 \mathrm{~V}(\mathrm{D}=50 \%)$

Fig. 12: Comparison between the measurement and simulation on $R_{\mathrm{DS} \text { (on) }}$ values when device switches at different voltages

\section{B. Switching voltage at $120 \mathrm{~V}$ and $100 \mathrm{~V}$}

The device is then switched at $120 \mathrm{~V}$, of which the comparison between the measurement and simulation results is shown in Fig. 12a. Despite some difference between the measurement and simulation, the increase trend of the $R_{\mathrm{DS}(\mathrm{on})}$ values is represented well by the model. The bigger $R_{\mathrm{DS}(\text { on) }}$ values ripper in the simulation than in measurements is mainly relied on difference illustrated in Fig. 9 between the model and the measurement on $R_{\mathrm{DS}(\mathrm{on})}$ change influenced by detrapping time, where the model represents a bigger $R_{\mathrm{DS}(\text { on) }}$ value variation than the measurement.

The device is then switched at $100 \mathrm{~V}$, of which the comparison between the measurement and simulation results is shown in Fig. 12b. In this condition, parameters $R_{\mathrm{nd}}, R_{\mathrm{nt}}$ and $C_{\mathrm{n}}$ are the same as that shown in TABLE II, and parameters $V_{\mathrm{n}}$ is interpolated linearly based on the results obtained at TABLE II and TABLE III. It is shown in Fig. 12b that the simulation represents well the measurement, and obtained dynamic $R_{\mathrm{DS}(\text { on) }}$ values in this condition is between the values when device biased at $120 \mathrm{~V}$ and $80 \mathrm{~V}$.

Even with some difference, all the above results could validate the proposed model, which could be used to estimate device conduction loss including trapping effect in power converters at different switching voltages.

\section{CONCLUSiON}

In this paper, dynamic ON-state resistance $\left(R_{\mathrm{DS}(\mathrm{on})}\right)$ values of a commercial GaN-HEMT is measured at different bias voltages by a proposed electrical circuit, which is constituted by a voltage bias circuit to control DUT trapping time and a voltage clamping circuit to measure DUT on-state voltage. Compared to other electrical characterisation circuits, this circuit has less electrical components and could be easily implemented.

It is shown in the characterisation results that both trapping and detrapping time could influence device dynamic $R_{\mathrm{DS}(\text { on) }}$ values and a higher bias voltage would give rise to a higher dynamic $R_{\mathrm{DS}(\text { on) }}$ value. Based on the characterisation results, a behavioural model, which is constituted by a series of RC network, is proposed to represent device dynamic $R_{\mathrm{DS}(\text { on) }}$ values of different bias voltages. The model is then implemented in PSPICE and validated with the measurement when device switches in a power converter with different duty cycles and switching voltages.

Both the measurement and simulation results show that device $R_{\mathrm{DS}(\mathrm{on})}$ values would increase due to the trapping effects. The trapping effects of the device might still increase its $R_{\mathrm{DS} \text { (on) }}$ values in more than $100 \mathrm{kHz}$ high frequency power converters, because device detrapping time to reach its steadystate $R_{\mathrm{DS}(\text { on) }}$ value is longer than trapping time to increase its dynamic $R_{\mathrm{DS}(\text { on) }}$ values. The proposed model could be thus used to estimate device conduction loss in a power converter when using GaN-HEMT of different switching voltages.

\section{ACKNOWLEDGMENT}

The authors would like to thank UK Engineering and Physical Sciences Research Council (EPSRC) for the financial support under EP/K014471/1 and all the partners of the project Silicon Compatible GaN Power Electronics for technical discussions.

\section{REFERENCES}

[1] T. Mizutani, Y. Ohno, M. Akita, S. Kishimoto, and K. Maezawa, "A study on current collapse in AlGaN/GaN HEMTs induced by bias stress," Electron Devices, IEEE Transactions on, vol. 50, no. 10, pp. 2015-2020, 2003.

[2] M. Meneghini, D. Bisi, D. Marcon, S. Stoffels, M. Van Hove, T.-L. Wu, S. Decoutere, G. Meneghesso, and E. Zanoni, "Trapping and reliability assessment in d-mode gan-based mis-hemts for power applications," Power Electronics, IEEE Transactions on, vol. 29, no. 5, pp. 2199-2207, 2014.

[3] B. Lu, T. Palacios, D. Risbud, S. Bahl, and D. Anderson, "Extraction of Dynamic On-Resistance in GaN Transistors: Under Soft- and HardSwitching Conditions," in Compound Semiconductor Integrated Circuit Symposium (CSICS), 2011 IEEE, pp. 1-4, Oct 2011.

[4] D. Jin and J. Del Alamo, "Methodology for the Study of Dynamic ON-Resistance in High-Voltage GaN Field-Effect Transistors," Electron Devices, IEEE Transactions on, vol. 60, pp. 3190-3196, Oct 2013.

[5] G. Cao, A. Ansari, and H.-J. Kim, "A New Measurement Circuit to Evaluate Current Collapse Effect of GaN HEMTs Under Practical Conditions," Instrumentation and Measurement, IEEE Transactions on, vol. 64, pp. 1977-1986, July 2015. 
[6] R. Gelagaev, P. Jacqmaer, and J. Driesen, "A Fast Voltage Clamp Circuit for the Accurate Measurement of the Dynamic ON-Resistance of Power Transistors," Industrial Electronics, IEEE Transactions on, vol. 62, pp. 1241-1250, Feb 2015.

[7] R. Chu, A. Corrion, M. Chen, R. Li, D. Wong, D. Zehnder, B. Hughes, and K. Boutros, "1200-V Normally Off GaN-on-Si Field-Effect Transistors With Low Dynamic on -Resistance," Electron Device Letters, IEEE, vol. 32, pp. $632-634$, may 2011.

[8] J. Bocker, H. Just, O. Hilt, N. Badawi, J. Wurfl, and S. Dieckerhoff, "Experimental analysis and modeling of GaN normally-off HFETs with trapping effects," in Power Electronics and Applications (EPE'15 ECCEEurope), 2015 17th European Conference on, pp. 1-10, Sept 2015. 